\title{
Efficacy of two calcium phosphate pastes on the remineralization of artificial caries: a randomized controlled double-blind in situ study
}

\author{
Somkamol Vanichvatana and Prim Auychai
}

To test the efficacy of two calcium phosphate pastes compared to that of fluoride toothpaste on remineralizing artificial caries in situ, this study had a double-blind crossover in situ design, involving three experimental phases of 14 days each, with an 8-day washout period between phases. Nine healthy subjects participated in the study. The subjects wore removable palatal appliances mounted with six human enamel slabs with artificial caries lesions, and in each of the experimental phases, used one of the following methods two times/day: group A, brushing with $1.0 \mathrm{~g}$ of Colgate Regular Flavor, followed by applying $0.25 \mathrm{~g}$ of Tooth Mousse Plus; group B, brushing with $0.25 \mathrm{~g}$ of Clinpro Tooth Crème; and group C, brushing with $1.0 \mathrm{~g}$ of Colgate Regular Flavor. After 14 days, the enamel slabs (54 slabs/ group) were embedded in resin, sectioned and examined with a polarized-light microscope, and the lesion areas were quantified using Image-Pro Plus. All experimental groups showed a significant reduction in lesion area compared to the initial lesion area (paired $t$-test, $\boldsymbol{P}<0.001)$. The mean reduction in lesion area of Groups A, B and C were $(0.029 \pm 0.010),(0.030 \pm 0.009)$ and $(0.027 \pm 0.009) \mathrm{mm}^{2}$, respectively. There were no statistical differences between groups (Kruskal-Wallis test, $\boldsymbol{P}>0.05$ ). All three groups remineralized the enamel slab lesions, indicating model sensitivity to fluoride. Given the differences in usage amounts and treated regimens, Clinpro Tooth Crème provided similar benefits to the fluoride toothpaste; however, no additional benefit of Tooth Mousse Plus was observed when used in conjunction with the fluoride toothpaste.

International Journal of Oral Science (2013) 5, 224-228; doi:10.1038/ijos.2013.67; published online 13 September 2013

Keywords: Clinpro; casein phosphopeptide-amorphous calcium phosphate; fluoride; functionalized tricalcium phosphate; remineralization

\section{INTRODUCTION}

A better understanding of the oral environment and cariology has resulted in extensive changes in caries management towards a more conservative approach. The development of a caries lesion is now recognized as an imbalance in the demineralization and remineralization cycle. ${ }^{1}$ Many researchers are now investigating the use of techniques to induce remineralization of the affected tooth structure. The use of fluoride is an effective method for promoting the remineralization of an early lesion in enamel through the formation of fluorapatite. However, for every two fluoride ions, ten calcium ions and six phosphate ions are required to form one unit cell of fluorapatite $\left(\mathrm{Ca}_{10}\left(\mathrm{PO}_{4}\right)_{6} \mathrm{~F}_{2}\right){ }^{2}$ Hence, when topically applying fluoride, an inadequate amount of available calcium and phosphate ions can limit net enamel remineralization. ${ }^{2}$ Another option to enhance remineralization is to supply calcium and phosphate, and to deliver fluoride more effectively.

Two calcium phosphate compounds that can induce remineralization have been developed. Casein phosphopeptide-amorphous calcium phosphate (CPP-ACP) consists of casein phosphopeptide (CPP) aggregated with calcium phosphate to form clusters of amorphous calcium phosphate (ACP). This aggregation prevents calcium phosphate precipitation, resulting in a state of supersaturation with respect to enamel, preventing demineralization and promoting remineralization. ${ }^{3} \mathrm{CPP}-\mathrm{ACP}$ has been demonstrated to have anticariogenic activity in in vitro, animal and human in situ experiments, including a randomized controlled clinical trial. ${ }^{4} \mathrm{CPP}-\mathrm{ACP}$ is available as a professional dental product (GC Tooth Mousse; GC Corporation, Tokyo, Japan). GC Tooth Mousse containing 0.09\% fluoride is available as a CPP-ACPF paste (GC Tooth Mousse Plus; GC Corporation, Tokyo, Japan). CPP-ACPF has been reported to have a greater potential for remineralization than CPP-ACP. ${ }^{5}$ Another calcium phosphate compound is functionalized tricalcium phosphate $(f \mathrm{TCP})$, which is produced by the solid-state ball milling of beta-tricalcium phosphate and sodium lauryl sulfate. $f$ TCP can prevent calcium from prematurely interacting with ionic fluoride and forming calcium fluoride, thus delivering more fluoride and calcium ions to the enamel surface. ${ }^{6-7} f$ TCP has been shown to have remineralizing effects in both in vitro and in situ studies. ${ }^{8-10} f$ TCP has been commercially developed, and is now available as a $950 \mathrm{ppm}$ fluoride toothpaste (Clinpro Tooth Crème; 3M ESPE, Saint Paul, MN, USA).

The aim of our study was to compare the efficacy of CPP-ACPF and $f$ TCP (calcium phosphate pastes) with that of conventional $0.1 \%$ 
fluoride toothpaste in remineralizing enamel artificial caries lesions in a randomized, controlled, double-blind, crossover in situ trial.

\section{MATERIALS AND METHODS}

Preparation of artificial caries lesions and intra-oral appliance fabrication

Freshly extracted human premolar teeth were collected and sterilized with ethylene oxide gas for $18 \mathrm{~h}$. The minimum number of specimens required for the study was determined based on our pilot study. We calculated that we would need at least 52 specimens per group to give a two-sided 5\% significance level and a power of $80 \%$. Fifty-four teeth without caries, cracks or enamel malformation were selected and cleaned with pumice to remove debris/stains from the enamel surface. Artificial caries lesions were created by immersing each tooth in demineralization solution $\left(80 \mathrm{mg} \cdot \mathrm{L}^{-1}\right.$ carbopol, $50 \mathrm{mg} \cdot \mathrm{L}^{-1}$ hydroxyapatite, $85 \%$ lactic acid, $6 \mathrm{~mol} \cdot \mathrm{L}^{-1}$ sodium hydroxide, $\mathrm{pH} 4.8$ ), for 21 days at room temperature to produce lesions approximately 100 $150 \mu \mathrm{m}$ deep. After demineralization, a low-speed cutting machine (ISOMET 1000; Buehler, Lake Bluff, IL, USA) was used to vertically section the proximal surfaces of each tooth into two enamel slabs approximately $2 \mathrm{~mm}$ long $\times 1 \mathrm{~mm}$ wide $\times 2 \mathrm{~mm}$ thick. The four enamel slabs from each tooth were randomly assigned to either the initial lesion group or one of the three test groups.

Removable acrylic palatal appliances were individually fabricated for each subject. The components of the appliances consisted of a labial arch and two Adam's clasps. Two troughs $(7 \mathrm{~mm}$ long, $5 \mathrm{~mm}$ wide and $3 \mathrm{~mm}$ deep) were bilaterally cut into the acrylic base, parallel to the posterior teeth. Three enamel slabs were retained in each of the troughs by flowable composite resin to produce a $1 \mathrm{~mm}$ deep trough above the enamel surface to allow the establishment of plaque (Figure 1). All appliances were sterilized with ethylene oxide prior to intra-oral application.

\section{Experimental protocol}

All study protocols were approved by the Ethics Committee of the Faculty of Dentistry, Chulalongkorn University. The study was conducted in Bangkok, Thailand from 2010 to 2011. Nine healthy adults (eight females and one male) with an average age of $19 \pm 7$ years participated in our study. After gaining informed written consent, the subjects were given a thorough intra-oral examination and completed a medical history questionnaire. The study inclusion criteria consisted of having at least 22 teeth without clinically active caries, periodontal disease or other oral pathology. The subjects' mean unstimulated

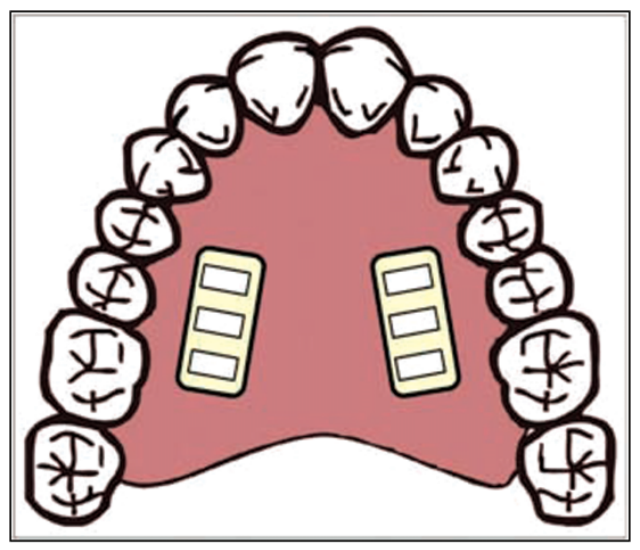

Figure 1 The removable acrylic appliance. salivary flow rate was $(0.8 \pm 0.3) \mathrm{mL} \cdot \mathrm{min}^{-1}$, and their mean stimulated salivary flow rate was $(3.7 \pm 0.8) \mathrm{mL} \cdot \mathrm{min}^{-1}$.

This double-blind, randomized, controlled in situ study comprised three distinct phases lasting 14 days each, which were preceded by an 8-day washout period to eliminate any residual effects of the previous product (Figure 2). The three products used were: (i) Tooth Mousse Plus (GC Corporation, Tokyo, Japan); (ii) Clinpro Tooth Crème (3M ESPE, Saint Paul, MN, USA); and (iii) $0.1 \%$ fluoride toothpaste (Colgate Regular Flavor; Colgate-Palmolive, Bangkok, Thailand). The three products were given to the subjects contained in syringes and were identical in appearance.

On the last day of the washout periods, the subjects wore the appliances without using any products to check for any irritation (day before the first phase only) and to allow for plaque accumulation. The assignment of the test products was based on a randomization scheme (Figure 3). We used a computer-generated list of random numbers for the sequential allocation of the subjects into the groups. Subject nos. 1-3 began as group A, subject nos. 4-6 began as group B and subject nos. 7-9 began as group C. All subjects were asked to maintain their normal dietary habits. The participants were instructed to wear the appliances for at least $12 \mathrm{~h}$ a day, including during eating and drinking. The use of any other oral hygiene product was prohibited. The test pastes were applied twice a day, after breakfast and dinner, based on the manufacturers' directions. For group A, the

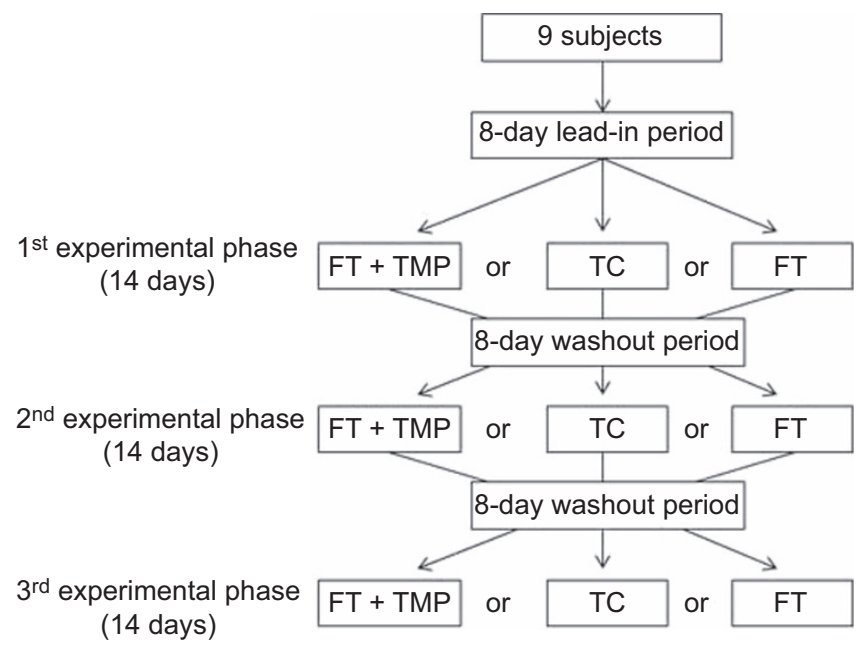

Figure 2 The study protocol. FT, 0.1\% fluoride toothpaste; TMP, Tooth Mouse Plus; TC, Clinpro Tooth Crème.

Randomized

$(n=9)$

Phase 1

$A, A, A, B, B, B, C, C, C$

Phase 2

$A B, A B, A C, B A, B A, B C, C A, C A, C B$

Phase 3

$A B C, A B C, A C B, B A C, B A C, B C A, C A B$, $\mathrm{CAB}, \mathrm{CBA}$

Figure 3 The randomization scheme of the subjects. A, 0.1\% fluoride toothpaste+Tooth Mousse Plus; B, Clinpro Tooth Crème; C, 0.1\% fluoride toothpaste. 


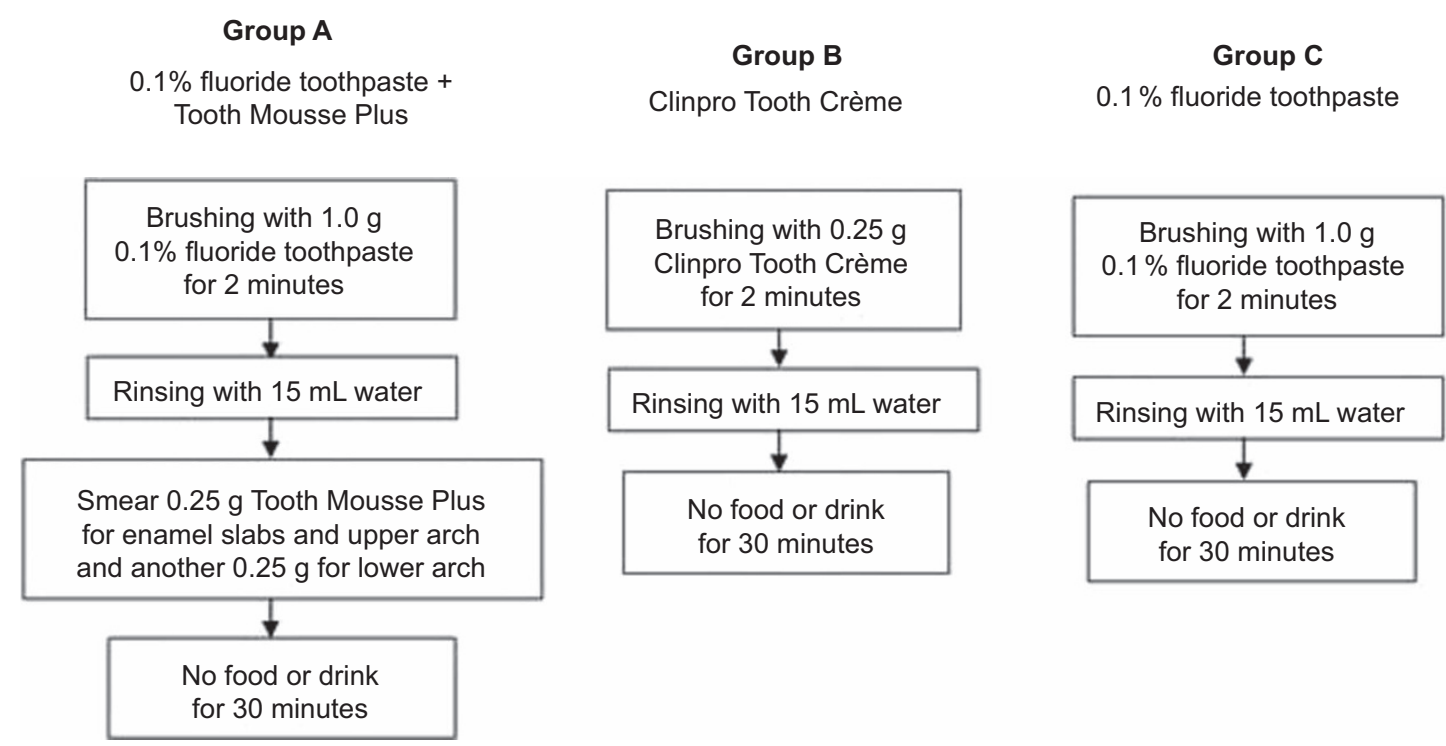

Figure 4 Application method of each group.
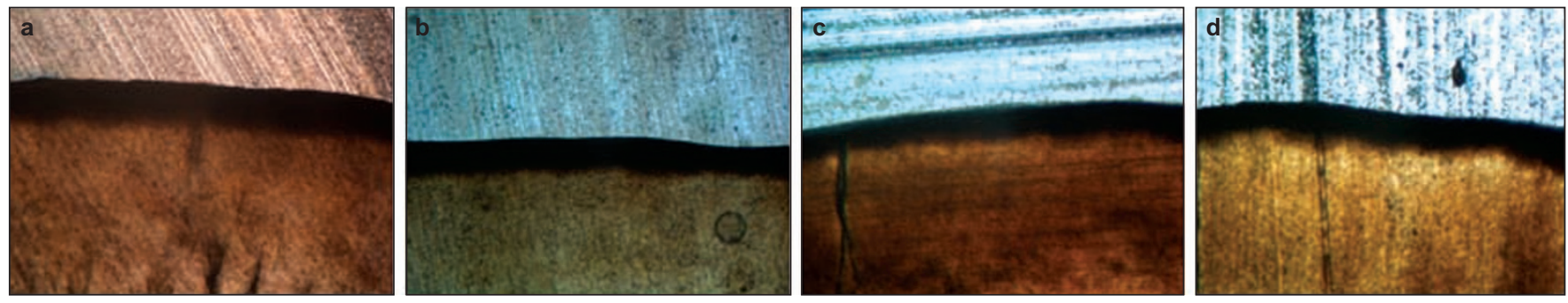

Figure 5 Lesions viewed with a polarized light microscope. (a) Initial lesion group; (b) Group A, 0.1\% fluoride toothpaste+ Tooth Mousse Plus; (c) Group B, Clinpro Tooth Crème; (d) Group C, 0.1\% fluoride toothpaste.

subjects brushed their teeth with $1.0 \mathrm{~g}$ of fluoride toothpaste for $2 \mathrm{~min}$ while wearing their appliances and taking care not to brush the slabs, then expectorated the slurry and rinsed their mouths with $15 \mathrm{~mL}$ of tap water for $10 \mathrm{~s}$. After brushing, the subjects squeezed $0.25 \mathrm{~g}$ of Tooth Mousse Plus onto their fingers and applied it on the enamel slabs and their upper teeth. The subjects used another $0.25 \mathrm{~g}$ of Tooth Mousse Plus to apply on their lower teeth. For group B, the subjects were instructed to brush their teeth with $0.25 \mathrm{~g}$ of Clinpro Tooth Crème and then expectorated the slurry and gently rinsed their mouths with tap water. For group C, the subjects were instructed to use the same brushing method as group B, using $1.0 \mathrm{~g}$ of fluoride toothpaste. The subjects were instructed not to eat or drink for 30 min after any of the treatments (Figure 4). After the appliances had been worn for $12 \mathrm{~h}$, they were removed and cleaned with a toothbrush. The subjects were instructed to avoid brushing directly on the enamel slabs. After cleaning, the appliances were stored in sealed moist plastic containers at room temperature. At the end of each 14-day period, the enamel slabs were detached and kept in deionized water at room temperature until they were embedded in resin, and the appliances were stored in tap water at room temperature until the next phase. After the washout period, new enamel slabs were used for the next experimental phase. This procedure was repeated until the subjects had rotated through all three treatment groups.

\section{Sectioning and polarized light microscopy}

The enamel slabs were embedded in resin, and 100-150 $\mu \mathrm{m}$ sections were prepared using a microtome (Leica Sp 1600; Leica, Solms, Germany). The label containing the information of each slab: subject number, product used and specimen number were concealed and randomized, and the examiner was blinded to which treatment has been administered. Fifty-four specimens per group were examined with a polarized-light microscope (9300 Meiji; Meiji Techno, Chikumazawa, Japan) after imbibition in deionized water. Digital images were taken with a color video camera (Meiji CX 3800; Meiji Techno, Chikumazawa, Japan) (Figure 5) and were transformed into black-and-white images using Microsoft Office Picture Manager version 12.0.6412.1000. The lesion areas were measured using Image-Pro Plus version 4.5.0.29 (Media Cybernetics, Bethesda, USA). Three measurements were made for each specimen, and the average was determined.

\section{Data analysis}

Statistical analysis of the data was conducted using SPSS statistical software version 17.0 with significance $(\alpha)$ set at 0.05 . All data were examined for normality and homogeneity of variance. The pre- and post-test parameters within each group were compared using the paired $t$-test at the $95 \%$ confidence level. Intergroup comparisons were performed using the Kruskal-Wallis test $(P<0.05)$. 
Table 1 Mean lesion area, mean lesion area reduction and the percentage of lesion area reduction

\begin{tabular}{|c|c|c|c|c|}
\hline Groups ( $n=54)$ & Mean \pm s.d. $/ \mathrm{mm}^{2}$ & $\begin{array}{l}\text { Mean lesion area } \\
\text { reduction } \pm \text { s.d. } / \mathrm{mm}^{2}\end{array}$ & $\begin{array}{l}\text { Median lesion area reduction } \\
\quad \text { (interquartile) } / \mathrm{mm}^{2}\end{array}$ & Lesion area reduction/\% \\
\hline Initial lesions & $0.202 \pm 0.028$ & - & - & - \\
\hline $\begin{array}{l}\text { A (fluoride toothpaste }+ \text { Tooth } \\
\text { Mousse Plus) }\end{array}$ & $0.173 \pm 0.028$ & $0.029 \pm 0.010$ & $0.030(0.030-0.325)$ & 16.81 \\
\hline B (Clinpro Tooth Crème) & $0.172 \pm 0.029$ & $0.030 \pm 0.009$ & $0.030(0.020-0.040)$ & 17.31 \\
\hline C (fluoride toothpaste) & $0.175 \pm 0.029$ & $0.027 \pm 0.009$ & $0.030(0.020-0.030)$ & 15.45 \\
\hline
\end{tabular}

\section{RESULTS}

All participants completed the study with $100 \%$ compliance. No adverse events were reported by the participants. The enamel caries lesion data for the study are presented in Table 1. After 14 days of use in $s i t u$, each product produced a statistically significant reduction in lesion area $(P<0.05)$ compared to the initial lesion group. However, we found no significant differences between the three test groups $(P>0.05)$.

\section{DISCUSSION}

The results of the present study demonstrate that both CPP-ACPF paste and sodium fluoride paste containing functionalized tricalcium phosphate can enhance the remineralization of artificial caries lesions in enamel when used in situ. However, when comparing the remineralization induced by these two calcium phosphate pastes with that of regular $0.1 \%$ fluoride toothpaste, no statistical differences were found. The results of our study are inconsistent with a prior study conducted by Shen et al., ${ }^{11}$ where CPP-ACPF induced significantly more remineralization compared to $0.1 \%$ fluoride toothpaste. This may be because in their study, CPP-ACPF paste was applied four times a day in the form of a slurry made from $1 \mathrm{~g}$ of CPP-ACPF in $4 \mathrm{~mL}$ of distilled deionized water. In contrast, in the present study, the participants used Tooth Mousse Plus following the manufacturer's instructions that recommended using a pea-size amount (approximately $0.25 \mathrm{~g}$ ) of Tooth Mousse Plus on each arch after brushing with fluoride toothpaste. These differences in application methods may alter the remineralization effect of the product. In the study of Shen et al., ${ }^{11}$ the participants received a greater amount of calcium, phosphate and fluoride. Similarly, the increased frequency of CPP-ACPF use may have affected the results of their study. Rinsing with CPP-ACPF slurry four times daily likely allowed more CPP-ACPF to accumulate in the subjects' dental plaque and saliva compared with the present study. Moreover, the participants in Shen et al.'s ${ }^{11}$ study also received fluoride from their normal oral hygiene procedures (brushing with $0.1 \%$ fluoride toothpaste). This may have resulted in their $\mathrm{CPP}-\mathrm{ACPF}$ group demonstrating a greater remineralizing effect. However, in the present study, we chose application methods simulating typical product use, rather than equal amounts of each product, the results of which could be expected to reflect what would occur clinically.

Although studies have indicated that $\mathrm{CPP}-\mathrm{ACP}$ paste has a remineralization effect, ${ }^{12-15}$ the results of the current study did not show any additional effect of $\mathrm{CPP}-\mathrm{ACP}$ on the remineralization of artificial caries when used as a supplement to regular fluoride toothpaste. These findings are likely because the prior studies used protocols that were different from the manufacturer's instruction, which the present study employed. In the prior studies, specimens were immersed in diluted CPP-ACP paste ${ }^{12}$ or were applied with CPP-ACP paste for $30 \mathrm{~min},{ }^{14}$ which could enhance remineralization more than using the paste following the manufacurer's instructions. In contrast, the results of the present study were consistent with a clinical study by Bröchner et al., ${ }^{16}$ which compared subjects using $\mathrm{CPP}-\mathrm{ACP}$ as a supplement to daily fluoride toothpaste with subjects who brushed with fluoride toothpaste alone. Bröchner et al. ${ }^{16}$ showed that the remineralization was not superior to the natural regression following daily use of fluoride toothpaste. Thus, based on our results, using CPP-ACP could make daily oral hygiene procedures more complicated and increase costs.

The results of our study showed no significant difference in remineralization between Clinpro Tooth Crème and regular $0.1 \% \mathrm{~F}$ toothpaste. However, the participants used Clinpro Tooth Crème in only onefourth the amount $(0.25 \mathrm{~g})$ of the regular toothpaste. These findings agree with a previous study by Mensinkai et al. ${ }^{10}$ where $0.05 \%$ fluoride toothpaste containing $f$ TCP tended to produce greater remineralization compared to $0.05 \%$ fluoride toothpaste when used in the same amounts. The subjects in our study followed the manufacturer's instructions and used Clinpro Tooth Crème in pea-size amounts $(0.25 \mathrm{~g})$ and received a lower amount of fluoride, but we saw the same remineralizing effect as was seen with using $1.0 \mathrm{~g}$ of $0.1 \%$ fluoride toothpaste or fluoride toothpaste followed by CPP-ACPF paste. As a result, Clinpro Tooth Crème may be of benefit for patients who want to avoid using fluoride or for children at risk of developing fluorosis.

Our investigation also shows that regular $0.1 \%$ fluoride toothpaste is effective in the remineralization of artificial caries in situ. Fluoride toothpaste is an effective product for the control of dental caries and can be used by the majority of the population. Brushing with fluoride toothpaste is an easy procedure that is low in cost, and this product is readily available. Indeed, the evidence on the effect of fluoride toothpaste on the prevention of dental caries has been extensively reviewed. ${ }^{17-18}$ Therefore, dentists should advise patients to use fluoride toothpaste, especially in terms of cost-effectiveness.

An in situ model is an appropriate method for the evaluation of the anticaries potential of new compounds added to dentifrices or pastes. There are many advantages to in situ studies that are performed in the human mouth, in contrast to in vitro or animal studies. In situ studies allow the control of experimental variables and a flexibility of experimental design that is impossible to achieve in clinical trials. However, this type of study depends heavily on compliance by the test subjects. ${ }^{19}$ To increase the subject's compliance, we gave them an instruction checklist and directed them to check off each step as it was completed. In addition, we randomly called the subjects during the experiment to remind them to use the products.

To provide a scientifically supported clinical recommendation for the use of the products examined in our investigation, further study should examine a larger population, include a group of high caries risk participants (e.g., have active caries lesions or low salivary flow rate), have a longer treatment time and include additional remineralizing products such as a $0.05 \%$ fluoride toothpaste. Moreover, additional studies should include a paste without therapeutic ingredients, which would allow for a better delineation between the effect of these pastes and saliva only, and alternative methods of using these products. For example, using CPP-ACPF 
paste 1-2 $\mathrm{h}$ after brushing with fluoride toothpaste may permit the discrimination of the remineralizing effect of CPP-ACPF from that of fluoride toothpaste.

In conclusion, all three groups remineralized the enamel slab lesions, indicating model sensitivity to fluoride. Given the differences in usage amounts and treated regimens, Clinpro Tooth Crème provided similar benefits to the $0.1 \%$ fluoride toothpaste, however, no additional benefit of Tooth Mousse Plus was observed when used in conjunction with the $0.1 \%$ fluoride toothpaste.

\section{ACKNOWLEDGEMENTS}

This study was supported by the Dental Research Fund, Faculty of Dentistry, Chulalongkorn University. The authors wish to thank Dr Kevin Tompkins for his kind assistance in editing the paper and Miss Paipan Pitayanon for her statistical analysis advice. Publication of this manuscript is supported by Open Fund of State Key Laboratory of Oral Diseases, Sichuan University.

1 Featherstone JD. Dental caries: a dynamic disease process. Aust Dent J 2008; 53(3): 286-291.

2 Reynolds EC, Walsh LJ. Additional aids to the remineralisation of tooth structure// Mount GJ, Hume WR. Preservation and restoration of tooth structure. 2nd ed. Brisbane: Knowledge Books and Software, 2005: 111-118.

3 Reynolds EC, Cai F, Cochrane NJ et al. Fluoride and casein phosphopeptideamorphous calcium phosphate. J Dent Res 2008; 87(4): 344-348.

4 Reynolds EC. Casein phosphopeptide-amorphous calcium phosphate: the scientific evidence. Adv Dent Res 2009; 21(1): 25-29.

5 Reynolds EC, Cai F, Shen $\mathrm{P}$ et al. Retention in plaque and remineralization of enamel lesions by various forms of calcium in a mouthrinse or sugar-free chewing gum. J Dent Res 2003; 82(3): 206-211.

6 Karlinsey RL, Mackey AC, Walker ER et al. Surfactant-modified beta-TCP: structure, properties, and in vitro remineralization of subsurface enamel lesions. J Mater Sci Mater Med 2010; 21(7): 2009-2020.
7 Karlinsey RL, Pfarrer AM. Fluoride plus functionalized $\beta$-TCP: a promising combination for robust remineralization. Adv Dent Res 2012; 24(2): 48-52.

8 Karlinsey RL, Mackey AC, Stookey GK. In vitro remineralization efficacy of NaF systems containing unique forms of calcium. Am J Dent 2009; 22(3): 185-188.

9 Karlinsey RL, Mackey AC, Stookey GK et al. In vitro assessments of experimental NaF dentifrices containing a prospective calcium phosphate technology. Am J Dent 2009; 22(3): 180-184.

10 Mensinkai PK, Ccahuana-Vasquez RA, Chedjieu I et al. In situ remineralization of white-spot enamel lesions by 500 and 1,100 ppm F dentifrices. Clin Oral Investig 2012; 16(4): 1007-1014.

11 Shen P, Manton DJ, Cochrane NJ et al. Effect of added calcium phosphate on enamel remineralization by fluoride in a randomized controlled in situ trial. J Dent 2011; 39(7): 518-525.

12 Yamaguchi K, Miyazaki M, Takamizawa T et al. Effect of CPP-ACP paste on mechanical properties of bovine enamel as determined by an ultrasonic device. J Dent 2006; 34(3): 230-236.

13 Rahiotis C, Vougiouklakis G. Effect of a CPP-ACP agent on the demineralization and remineralization of dentine in vitro. J Dent 2007; 35(8): 695-698.

14 Ogata K, Warita S, Shimazu K et al. Combined effect of paste containing casein phosphopeptide-amorphous calcium phosphate and fluoride on enamel lesions: an in vitro $\mathrm{pH}$-cycling study. Pediatr Dent 2010; 32(5): 433-438.

15 Hegde MN, Moany A. Remineralization of enamel subsurface lesions with casein phosphopeptide-amorphous calcium phosphate: a quantitative energy dispersive $\mathrm{X}$ ray analysis using scanning electron microscopy: an in vitro study. J Conserv Dent 2012; 15(1): 61-67.

16 Bröchner A, Christensen C, Kristensen B et al. Treatment of post-orthodontic white spot lesions with casein phosphopeptide-stabilised amorphous calcium phosphate. Clin Oral Investig 2011; 15(3): 369-373.

17 Wong MC, Clarkson J, Glenny AM et al. Cochrane reviews on the benefits/risks of fluoride toothpaste. J Dent Res 2011; 90(5): 573-579.

18 Rasines G. Fluoride toothpaste prevents caries in children and adolescents at fluoride concentrations of 1000 ppm and above. Evid Based Dent 2010; 11(1): 6-7.

19 Zero DT. In situ caries models. Adv Dent Res 1995; 9(3): 214-230.

This work is licensed under a Creative Commons Attribution-NonCommercial-NoDerivative Works 3.0 Unported License. To view a copy of this license, visit http:// creativecommons.org/licenses/by-nc-nd/3.0 